\title{
SELF-ASSEMBLING SINGLE CELLS ACROSS MICROPOSTS: FIRST AXIAL FORCE MEASUREMENTS IN IMMATURE CARDIOMYOCYTES
}

\author{
R.E. Taylor ${ }^{*}$, K. Kim, and B.L. Pruitt
}

Mechanical Engineering, Stanford University, Stanford, CA, USA

\begin{abstract}
We have designed and fabricated a novel silicone-based tool to enable measurement of force-generation by single immature heart cells. This tool combines micropost arrays with a sacrificial layer process to permit the self-assembly of developing cardiomyocytes across wide spread microposts. Here we report the first axial force-generation measurements of neonatal rat cardiomyocytes. This technique has critical utility for tissue engineers in evaluating immature phenotypes.
\end{abstract}

\section{INTRODUCTION}

Measurements of single immature heart cell mechanics has not been possible using the same axial methods that enable adult cardiomyocyte (CM) force-generation characterization. ${ }^{1,2,3}$ Current methods rely on the persistent elongated shape of isolated adult $\mathrm{CMs}$ and are not compatible with immature heart cells due to the spherical phenotype in dissociated, immature cells or stem cells. However, there is also a need to assay force generation in immature or stem cell derived CMs throughout development to create functional, active cardiac grafts.

Here we present a novel technique that enables such force measurements for the first time by combining microfabricated,force-sensing platforms with a cell-culture sacrificial layer technique. We fabricate PDMS-based devices and self-assemble single immature CMs across pairs of wide spread microposts. This low cost technique achieves massively parallel cell testing with the desired 2-point axial cell loading configuration and force fidelity with potentially higher adhesion strength than gold-standard carbon-fiber methods adult $\mathrm{CM}$ techniques (and without glue!).

\section{THEORY AND CALIBRATION}

Euler beam theory is suitable for modeling the small displacements of these high aspect ratio posts (Figure 1a). The elastic modulus, E, must be known to perform these calculations.

$$
F=\frac{3 E \pi r^{4}}{4 H^{3}} \Delta x
$$

Due to batch modulus variation, we instead calibrate our PDMS by deflecting the microposts with a characterized piezoresistive microcantiliver. ${ }^{4}$ Deviations from classical beam theory due to substrate deformation and viscoelastic effects as well as modulus variability are mitigated by this calibration (see Figure $1 \mathrm{~b}$ and $1 \mathrm{c}){ }^{5.6} \mathrm{We}$ calibrated 10 microposts and found $\mathrm{E}=1.23 \pm$ $0.19 \mathrm{MPa}$ and an average beam stiffness of $\mathrm{k}=0.0472 \pm 0.0074$ $\mathrm{N} / \mathrm{m}$ (Table 1).

Table 1: Modulus of elasticity, effective post stiffness, and peak force generation based upon calibration results.

\begin{tabular}{|l|c|}
\hline \multicolumn{2}{|c|}{ Calibration and Measurements } \\
\hline Effective post stiffness, $\mathrm{k}$ & $0.0472 \pm 0.0074 \mathrm{~N} / \mathrm{m}$ \\
\hline PDMS modulus of elasticity, $\mathrm{E}$ & $1.23 \pm 0.19 \mathrm{MPa}$ \\
\hline Peak cell force generation, $\mathrm{F}$ & $23.8 \pm 6.0 \mathrm{nN}$ \\
\hline
\end{tabular}
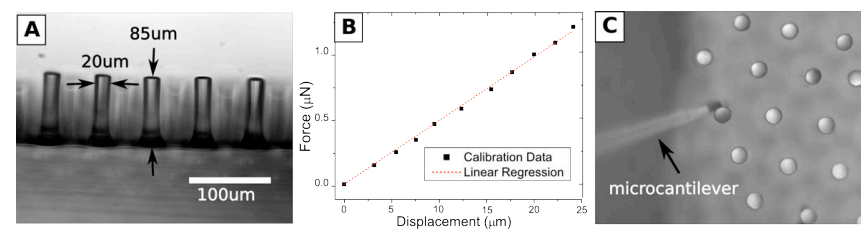

Figure 1: (A) Cylindrical posts are 20 $\mathrm{\mu m}$ in diameter and $85 \mu \mathrm{m}$ tall. Scale bar represents $100 \mu m$. (B) For small displacements force is linearly proportional to displacement. (C) Post stiffnesses are calibrated with piezoresistive microcantilevers.

\section{METHODS AND RESULTS}

The overall process flow (Figure 2) consists of four steps: post fabrication, sacrificial layer deposition, protein/cell deposition, and dissolution of sacrificial layer.

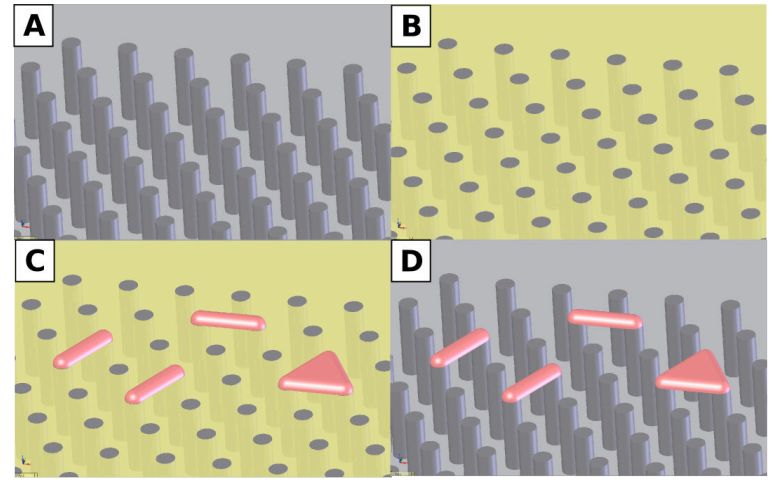

Figure 2: (A) Seeding begins with an $m P A D$ array that is (B) flooded with PNIPAAm to yield a smooth surface. Next, (C) cells spread across the flat surface of posts and sacrifical polymer, and (D) after 4-72 hours of attachment substrates are cooled to dissolve the PNIPAAm leaving cells suspended across posts.

\section{Post Fabrication}

Micropost array detectors (mPADs) made of polydimethylsiloxane (PDMS, Sylgaard 184) consist of $20 \mu \mathrm{m}$ diameter by $85 \mu \mathrm{m}$ high posts in a hexagonal array with $60 \mu \mathrm{m}$ spacing (Figure 2). mPADs are fabricated according to the protocol used by Tan et al. ${ }^{7}$

\section{Sacrificial Layer}

The thermo-responsive polymer poly( $\mathrm{N}$-isopropylacrylamide) (PNIPAAm, Polysciences) is used as a sacrificial layer. ${ }^{8}$ Posts are inverted on a soft 40:1 PDMS backing layer and flooded with 1\% aqueous PNIPAAm. Here we take advantage of the aqueous solution repulsion from the hydrophobic-hydrophobic stack to prevent PNIPAAm from coating the tops of the posts. Devices are evaporated overnight and the backing is peeled the next morning (Figure 3).

\section{Cell Seeding}

For all protein and cell coating steps, care is taken to judiciously maintain substrate temperature at $37^{\circ} \mathrm{C}$ to avoid 
PNIPAAm dissolution. Specific protein patterning is unnecessary since cells preferentially attach to the microposts. Devices are coated in $0.1 \%$ gelatin solution for $1 \mathrm{hr}$ followed by cell seeding and attachment for 2-3 days.

\section{Dissolve PNIPAAm}

When cells are adhered for testing, the devices are cooled to room temperature $(\sim 1$ hour $)$ to dissolve the PNIPAAm and release the posts (Figure 4).

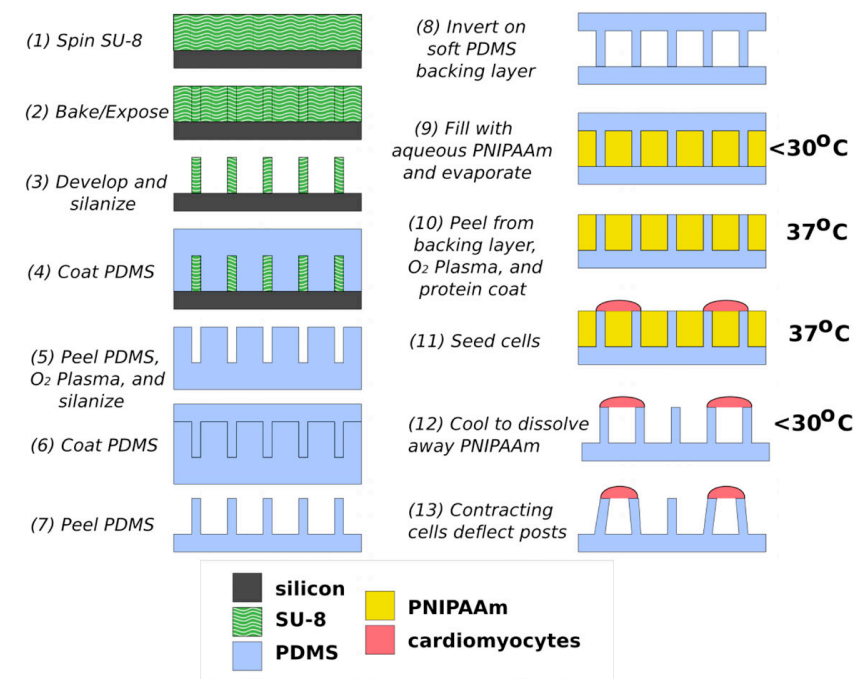

Figure 3: PDMS microposts are created by double-molding an SU-8 master post array. PDMS mPADs are inverted on a 40:1 PDMS backing layer creating a tight hydrophobic temporary bond. The sandwiched layers are filled with an aqueous $1 \%$ PNIPAAm solution. Once the PNIPAAm evaporates, the backing is peeled leaving a flat device with exposed post surfaces for subsequent cell attachment. Cells preferentially attach to the posts and therefore spread across posts without the need for protein patterning. Cooling causes the PNIPAAm to dissolve and leave behind single suspended cells.

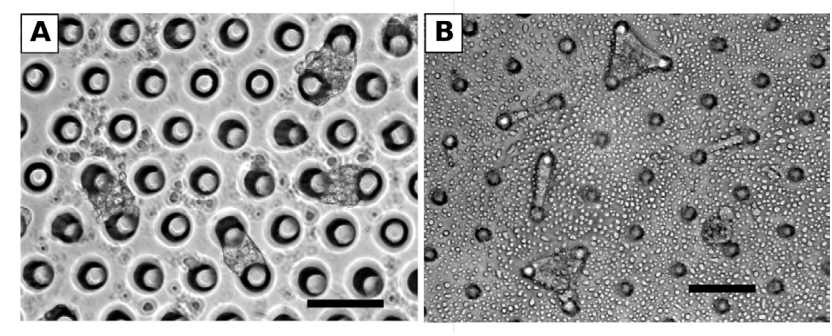

Figure 4: Beating cells successfully suspended across posts tend to take a linear form across two posts or a triangular form across three posts. Scale bar represents $100 \mu \mathrm{m}$. Cells that do not spread across posts remain associated with posts nonetheless $(B$, lower right).

\section{Cell measurements}

Cells are paced at $0.5 \mathrm{~Hz}$ and $1 \mathrm{~Hz}$ with a biphasic square wave of 15-25V (IonOptix Myopacer). Phase contrast microscopy video of cell contractions provides deflection data for force calculations (Figure 5). The peak force generated by rat neonatal cardiomyocytes is $23.8 \pm 6.0 \mathrm{nN}(\mathrm{n}=8)$. To our knowledge, such measurements have not been reported nor were possible before.
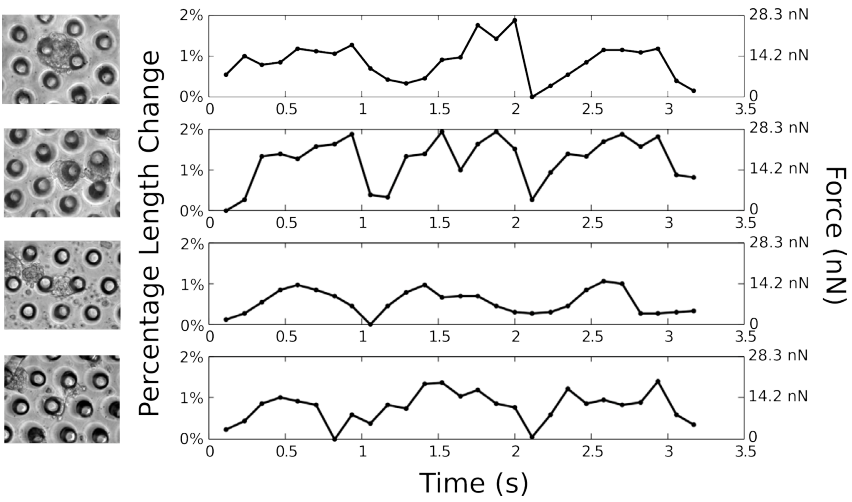

Figure 5: Percentage of cell length change and equivalent force generation vs time for four representative cells.

\section{ACKNOWLEDGMENTS}

This work has been performed with support from the following: NSF CAREER Award ECS-0449400, NSF EFRI-CBE 0735551, CIRM RC1-00151-1, NIH R21 HL089027, and Stanford Bio-X Grants. Microcantilevers were fabricated at the Stanford Nanofabrication Facility, which is supported by the NSF under grant 9731293 .

\section{REFERENCES}

[1] G. Iribe, M. Helmes, and P. Kohl, "Force-length relations in isolated cardiomyocytes subjected to dynamic changes in mechanical load," Am. J. Physiol. Heart Circ., 292, (2007) pp. H1487-H1497.

[2] G. Lin, S. J. Pister, K.P. Roos, "Surface Micromachined Polysilicon Heart Cell Force Transducer," JMEMS, 9, 1(2000) pp. 9-17.

[3] S. Yin, X. Zhang, C. Zhan, J. Wu, J. Xu, and J. Cheung, "Measuring single cardiac myocyte contractile force via moving a magnetic bead," Biophys. J., 88, 2(2005) pp. 14891495.

[4] S-J. Park, M.B. Goodman, and B.L. Pruitt. "Analysis of nematode mechanics by piezoresistive displacement clamp," PNAS, 104, 44(2007) pp. 17376-17381.

[5] I. Schoen, W. Hu, and V. Vogel, "Substrate Contributions in Elastic Pillar Arrays: Correction of Cellular Force Measurements", Biophysical Journal, 98, 3 (2010) p. 336a.

[6] I. Lin, K. Ou, Y. Liu, K. Chen, and X. Zhang, "Viscoelastic characterization and modeling of polymer transducers for biological applications," Journal of Microelectromechanical Systems, 18, 5(2009) pp. 1087-1099.

[7] J.L. Tan, J. Tien, D.M. Pirone, D.S. Gray, K. Bhadriraju, and C.S. Chen, "Cells lying on a bed of microneedles: an approach to isolate mechanical force," PNAS, 100, 4(2003) pp. 1484-1489.

[8] J.Z. Xi, J.J. Schmidt, and C.D. Montemagno, "Self-assembled microdevices driven by muscle", Nat. Mater., 4, (2005) pp. 180-184.

\section{CONTACT}

*R.E. Taylor, rebeccat@stanford.edu 